\title{
La azarosa supervivencia de una especie en un entorno agrícola fuertemente transformado Do: $10.153661 / 2201923.007$
}
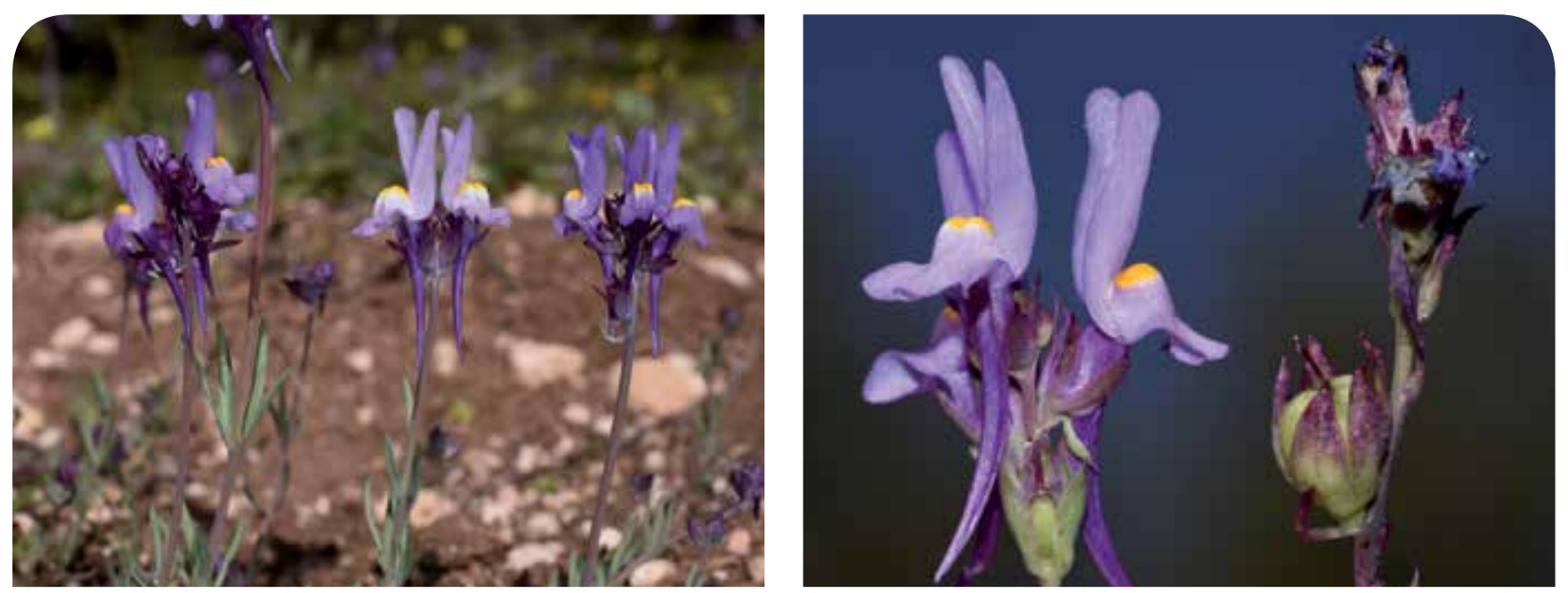

Figura 1. Aspecto general y detalle de Linaria qartobensis (Fotos: G. Blanca).

Era un día como cualquier otro, en el que los técnicos del LIFE Olivares Vivos se disponían a realizar el censo mensual de cubiertas vegetales de uno de los 20 olivares demostrativos que componen el proyecto, más concretamente en la provincia de Córdoba. Colocaron el cuadrado de 1x1 m cuidadosamente sobre el suelo del olivar e hicieron inventario de las plantas presentes en el mismo: "Leontodon longirostris, Calendula arvensis, Linaria amethystea... jespera, esta 'amethystea' es un poco rara, no me termina de convencer...! Perfecto, hagamos un pliego y un par de fotos al natural. Consultaremos a los expertos..." Ahí comenzó todo.

Ya en el Departamento de Botánica (Universidad de Granada) una identificación inicial, basada en Flora iberica (Sáez \& Bernal, 2009), conducía a considerar esta planta como Linaria ricardoi Cout., una especie del sur de Portugal que no se había recolectado en Andalucía y que además presenta flores y semillas mucho más pequeñas que las observadas en la nueva población cordobesa. Posteriormente, se comparó con otros taxones próximos de la sect. Supinae, particularmente con L. orbensis Carretero \& Boira, revelando constantes y apreciables diferencias tanto en caracteres florales como en la micromorfología de las semillas. La combinación original de toda una serie de caracteres con valor diagnóstico, el aislamiento con respecto a otras especies próximas y la imposibilidad de adscribirla a cualquier otra especie recogida en las floras regionales disponibles (Valdés et al., 1987; Sáez \& Bernal, 2009; Blanca et al., 2011), nos llevó a la conclusión de que se trataba de una nueva especie: L. qartobensis Blanca, Cueto, J. Fuentes, L. Sáez \& R. Tarifa.

\section{¿Una especie nueva en un sistema agrario?}

Parece una pregunta difícil de responder de modo afirmativo, más si cabe cuando el cultivo asociado es el olivar. A pesar de que podría ser uno de los agrosistemas con mayor potencial para retener diversidad al derivar de una especie autóctona (Olea europea var. sylvestris), en la actualidad el paisaje de olivar dista mucho de serlo. El proceso de industrialización que ha sufrido a lo largo de los años, en aras del aumento de la producción, se ha llevado por delante multitud de elementos que aportaban heterogeneidad al paisaje agrario, y por lo tanto diversidad. Uso indiscriminado de herbicidas, labranzas agresivas de la cubierta vegetal y eliminación de parches de vegetación natural son solo algunos de los ejemplos, fruto del proceso de homogeneización del paisaje y de la intensificación de las prácticas agrícolas. Un estudio reciente ha demostrado que la acción combinada de la intensificación de las prácticas agrarias con la simplificación del paisaje de olivar provoca la pérdida de una de cada tres especies arvenses del olivar andaluz (Rey et al., 2019). Por el momento, no se ha explorado de qué forma se ven afectadas las especies endémicas y/o protegidas frente a estas prácticas agrícolas dentro del olivar. Sin embargo, probablemente sean las más perjudicadas en este proceso. 
L. qartobensis ha sido sin duda una superviviente en este proceso de intensificación agraria y, aunque podría parecerlo, su hallazgo no ha sido fruto de la casualidad. No habría sido posible sin la realización de los exhaustivos muestreos de vegetación arvense llevados a cabo por el LIFE14 NAT/ ES/001094 Olivares Vivos (2015/2020). Este proyecto, financiado por la Unión Europea, cuenta con cuatro socios: SEO/BirdLife (socio coordinador), la Universidad de Jaén, la Estación Experimental de Zonas Áridas (CSIC, Almería) y la Diputación Provincial de Jaén. Actúan también como cofinanciadores la Fundación Patrimonio Comunal Olivarero y la interprofesional del Aceite de Oliva Español. Olivares Vivos es una iniciativa para incrementar la rentabilidad del olivar a partir de la recuperación de su biodiversidad; pretende crear un modelo de olivicultura rentable y sostenible, compatible con la conservación de la biodiversidad, con el objetivo final de crear una marca certificada para los aceites que gracias a su producción estén rescatando diversidad. En la actualidad se están ejecutando planes de restauración ambiental en los olivares, consistentes fundamentalmente en la gestión, mantenimiento y mejora de la cubierta vegetal, la restauración de zonas improductivas del olivar (plantación de especies vegetales herbáceas y leñosas creando o recuperando antiguos linderos y bosquetes y recuperando la red hídrica) y la mejora de la infraestructura de estos olivares (con la instalación de nidales de aves, insectos y murciélagos, postes nido para pequeñas y medianas rapaces, adecuación de balsas de riego, construcción de charcas y bebederos...), así como la utilización de prácticas agroecológicas más sostenibles. Para medir los efectos de la restauración ambiental se ha realizado un muestreo pre-operacional de vegetación arvense en los años 2016/2017, antes de la elaboración de las acciones de restauración, y uno post-operacional durante 2019/2020 tras la instalación de las mismas. Gracias a este diseño se podrá empezar a detectar la repercusión de estas acciones sobre la biodiversidad de arvenses, uno de los indicadores elegidos por el proyecto.

Durante este proceso se ha muestreado la vegetación arvense de un conjunto de 20 olivares demostrativos, distribuidos por toda la cuenca del Guadalquivir, una de las regiones oleícolas más importantes del mundo, obteniendo un total de 549 especies arvenses, casi un $13 \%$ de las especies vasculares de toda Andalucía, resultado que demuestra la capacidad del cultivo ecológico del olivar para albergar diversidad vegetal. Si tenemos en cuenta, además, que muchas de las fincas de olivar se encuentran conectando importantes áreas naturales protegidas de Andalucía (por ejemplo Parque Natural Sierras de Cazorla, Segura y las Villas, Parque Natural Sierra Mágina, Parque Natural de las Sierras Subbéticas cordobesas, etc.), nos podemos imaginar el potencial que podría llegar a tener este tipo de cultivo para conectar poblaciones de especies aisladas en la actualidad. De estas casi 550 especies, sin duda una ha sido la que se ha llevado todas las miradas, ya que además de ser bonita, L. qartobensis es una excelente embajadora de las buenas prácticas agrarias.

\section{Cuando la agricultura se alía con la protección de la flora}

La supervivencia de esta especie en el entorno donde se desarrolla debe considerarse absolutamente azarosa. Cuando la SEO/BirdLife de Andalucía contactó con uno de los autores de este artículo (C.G.) para la búsqueda de olivares a incluir

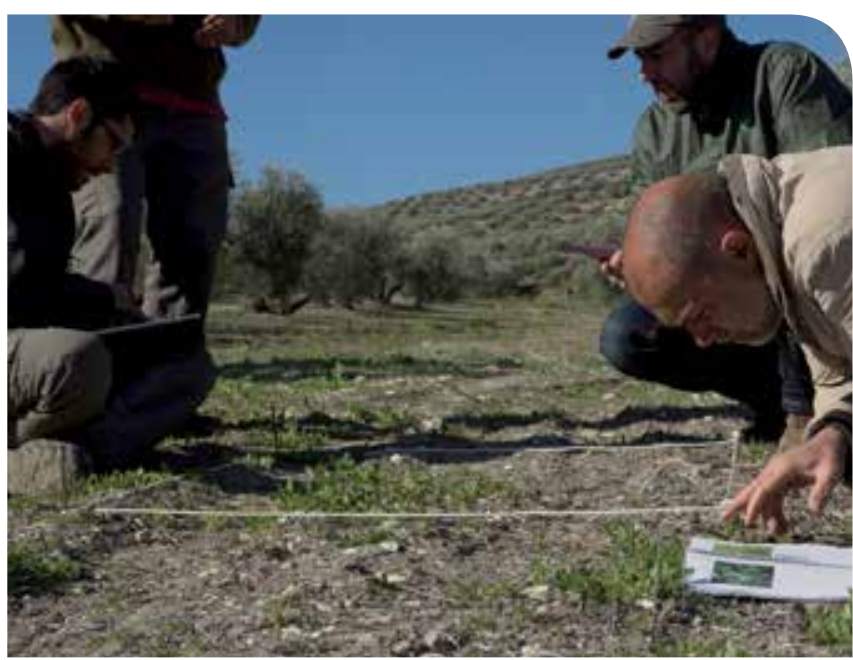

Figura 2. Grupo de investigadores censando plantas arvenses para el proyecto LIFE Olivares Vivos (Foto: LIFE Olivares Vivos).

en el proyecto Olivares Vivos en el sur de Córdoba, nadie podía sospechar las consecuencias que tendría la evaluación de la biodiversidad en algunos de ellos. Como miembro de una familia de olivicultores y productor de aceite bajo certificación ecológica, mostró con orgullo las labores realizadas durante los últimos años en la búsqueda del manejo adecuado de la biodiversidad en parcelas familiares, basado en la integración de comunidades herbáceas en las calles del olivar y la restauración de las zonas improductivas con arbustivas autóctonas.

Estos olivares, como casi todos los presentes en el paraje de los Llanos de Vanda, se plantaron por las generaciones que nos precedieron, después de desmontar las dehesas de encinas, quejigos y coscojas que antaño cubrieron las tierras de la Campiña Alta cordobesa. Primero se plantaron las tierras de menor calidad y más dificultad para ser trabajadas. Bancales y cerros se cubrieron con variedades locales tales como picudos, lechines, chorrúos y pajareros, entre otras variedades consideradas de "mala casta", que ofrecían una alta resistencia a las sequías estivales y buena adaptación a las tierras elegidas. Los llanos quedaron para el pastoreo extensivo, y las yeguadas, rebaños de cabras, ovejas, vacas y toros, aprovechaban los rastrojos y retazos de monte mediterráneo entre los olivares. Poco después, la extensión del olivar acabó con las tierras calmas de los Llanos de Vanda. El olivo picual se hizo dueño y señor de los olivares cordobeses, por su facilidad de recolección y grandes rendimientos de aceite.

Durante toda esta evolución, las técnicas de manejo fueron ecológicas, a pesar de la falta de un reconocimiento oficial, ya que las únicas herramientas disponibles por los agricultores eran las de la agroecología, que se basaba en el uso del arado para el control de las arvenses y el cobre para el control de las enfermedades criptogámicas de los olivares. Pero este escenario solo duró hasta la aparición de las nuevas tecnologías para la intensificación de la producción del olivar. Primero fue el tractor, con su gran capacidad para labrar y mover pesados aperos, y después los pesticidas, que provocaron un cambio radical en el paisaje de los olivares mediterráneos, y también de los cordobeses. Donde antes había comunidades de plantas herbáceas con collejas, tagarninas, tréboles, carretones, margaritas, amapolas, centaureas, corregüelas, viboreras y lenguazas, entre otras hierbas típicas de los olivares, y arbustivas como tomillos, aladiernos, cornicabras, lentiscos, jaras, 


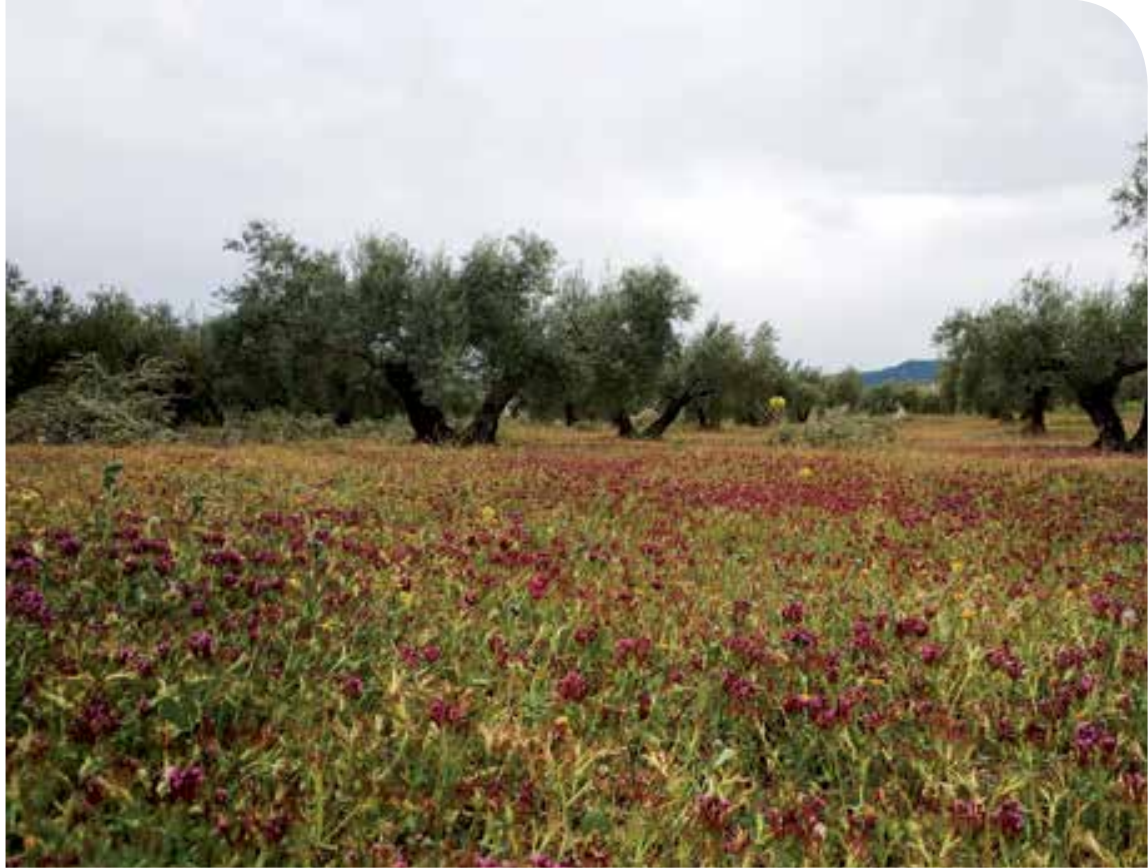

Figura 3. El cultivo del olivar alberga gran diversidad vegetal. Se han censado 549 especies arvenses en las parcelas estudiadas (Foto: LIFE Olivares Vivos).

madroños y olivillas, en la orla del bosque mediterráneo, solo se dejaron crecer árboles de olivo sobre un suelo desnudo y algunos retazos confinados en lindones y taludes.

Aun así, la naturaleza se defiende de las agresiones y busca los resquicios para sobrevivir. En esta ocasión la oportunidad vino dada por la resistencia de algunos agricultores a la intensificación del olivar y el mantenimiento de técnicas de manejo tradicionales en los olivares bajo sus cuidados. En ellos, los herbicidas tuvieron poca cabida y el control de las hierbas, hasta entonces naturales de los olivares, se siguió realizando mediante laboreo primero, y con las modernas desbrozadoras mecánicas después.

La casualidad quiso, además, que la parcela en la que se ha encontrado $L$. qartobensis haya estado cuidada desde hace tres generaciones por la familia Gálvez, impulsores de la producción ecológica de aceite en la comarca. Bajo este sistema de manejo, la zona donde se encuentra la población fue considerada como zona de reserva de biodiversidad, evitando el paso de maquinaria, manteniendo y potenciando la vegetación herbácea sin desbrozar hasta su senescencia, y conservando los usos y manejos tradicionales en la misma.

En este particular manejo de la parcela, hay que destacar algunos aspectos no relacionados necesariamente con el manejo bajo certificación ecológica. Uno es la ausencia de laboreo desde hace varias décadas en las zonas con mayor presencia de individuos de $L$. qartobensis, lo que podría indicar la falta de adaptación de la especie a los movimientos verticales de tierras, como los realizados con gradas y vertederas. Sin embargo, el uso de los vibradores de tronco, que provocan pequeños movimientos de tierra durante la operación de derribo de la aceituna, podría afectarle positivamente.

Otro aspecto a tener en cuenta es la relación entre la distribución espacial de la población, con las zanjas de drenaje que Antonio Gálvez hizo con el propósito de evitar el encharcamiento en la parcela. Inconscientemente, esto permitió mantener las condiciones ideales para la conservación de la especie, mientras que otras poblaciones presentes en lugares aledaños desaparecerían, con toda seguridad, por la intensificación del arado y el uso sistemático de los herbicidas.

Por último, el mantenimiento del volumen de copa de los olivos, optimizado para la producción de aceituna, ha debido contribuir positivamente a mantener una iluminación apropiada, tanto para conseguir las temperaturas necesarias para la germinación de las semillas en otoño, como para el desarrollo fenológico posterior bajo las temperaturas del invierno. Tampoco hay que olvidar la interacción de esta especie con otros organismos de los olivares. En este sentido, la falta de uso de insecticidas ha debido tener influencias positivas en la biota edáfica y en la polinización de las flores de la especie, aspecto que requerirá un estudio posterior, como el resto de su biología reproductiva.

Hoy continúa la presión sobre la población, por parte de los agricultores vecinos, a la espera de que las autoridades medioambientales establezcan las medidas adecuadas para preservar este precioso endemismo para las generaciones futuras.

\section{Aspectos botánicos}

Linaria sect. Supinae (Benth.) Wettst. es la sección taxonómicamente más compleja de todo el género, que se incluye en la tribu Antirrhineae Dumort. (Plantaginaceae). Está altamente diversificada en la cuenca mediterránea, siendo la península Ibérica su principal centro de diversidad. Muchas de las especies que incluye son endémicas de áreas relativamente reducidas, y a menudo muestran un comportamiento ecológico muy estricto, tales como $L$. accitensis Sáez et al. (depresión de Guadix, Granada, en substratos volcánicos), L. amoi Campo ex Amo (sierras de Tejeda y Almijara, limítrofes entre Granada y Málaga, en arenales y roquedos dolomíticos), $L$. argillicola Juan et al. (depresión de Guadix, Granada y zonas adyacentes de la provincia de Jaén, en substratos yesíferos), L. glacialis Boiss. (endémica de las cumbres de Sierra Nevada, Granada, en pedregales y roquedos esquistosos), L. platycalyx Boiss. (Serranía de Ronda, Málaga y Cádiz, en roquedos y pedregales calizos), L. saturejoides Boiss. (en roquedos y arenales dolomíticos o calizos de las provincias de Granada, Málaga y Almería) y L. verticillata Boiss. (montañas del sureste de la península Ibérica, en roquedos calizos o silíceos).

Linaria qartobensis, junto a L. ricardoi y L. orbensis, que son las especies más emparentadas, conforman un pequeño grupo de especies que habitan en cotas bajas, formando parte de pastizales efímeros asociados a campos de cultivo. Las diferencias morfológicas más importantes que separan a $L$. qartobensis de las otras dos especies mencionadas son la corola sin venas oscuras apreciables, flores y frutos de mayor tamaño, y semillas con el disco negro contrastando con el color pálido del ala y de los prominentes tubérculos del propio disco central, entre otros caracteres. 
Solo se conoce una población de L. qartobensis, perteneciente al municipio de Castro del Río (provincia de Córdoba), que incluye alrededor de 2.000 individuos reproductores, con una extensión de presencia y área de ocupación de $2 \mathrm{~km}$. Fuera de esta área, solo se han observado algunos individuos aislados distribuidos en zonas colindantes, no excediendo los $5 \mathrm{~km}$ de distancia de la población principal, por lo que debe considerarse como endemismo de distribución muy restringida. Se desarrolla en terrenos margosos propios de la depresión del Guadalquivir, en suelos desarrollados de tipo vertisol, entre cultivos de olivos, a una altitud de 300-400 m.

\section{Estado de conservación}

La única población conocida de L. qartobensis no está incluida en ninguna zona protegida, ni en algún Lugar de Interés Comunitario (LIC), por lo que queda fuera de cualquier área de la red Natura 2000. En la Comunidad Autónoma Andaluza, la Ley $8 / 2003$ de la flora y fauna silvestres, en su artículo 7.2.c establece la prohibición de "destruir, recoger, cortar, talar o arrancar, en parte o en su totalidad, especímenes naturales de la flora silvestre, así como destruir sus hábitats", aunque estas medidas tienen escasa repercusión y son muy difíciles de aplicar en la práctica. Además, el artículo 9 establece excepciones a estas prohibiciones previa autorización expresa de la Consejería competente en materia de medio ambiente.

Las causas potenciales de amenaza que pueden afectar a su supervivencia incluyen su limitada área de distribución, las prácticas agrícolas inadecuadas y, sobre todo, la aplicación de herbicidas. Utilizando las categorías y recomendaciones de IUCN (2012, 2017), L. qartobensis debe considerarse como especie 'en peligro crítico' $(C R)$, según los siguientes criterios: A1cd; B1ab(i,ii,iii,v)+2ab(i,ii,iii,v).

Entre las medidas recomendadas para su conservación se precisa un seguimiento de la única población conocida, mantenimiento de las actividades agrícolas respetuosas con la conservación de la biodiversidad y, en suma, un plan de manejo y conservación, al tiempo que debe ser incluida en las listas rojas de plantas vasculares establecidas tanto a nivel nacional como autonómico.

Tan solo tras la publicación de la Flora Vascular de Andalucía Oriental (Blanca et al., 2011), se han descrito más de 30 nuevos taxones en Andalucía, más de la mitad de ellos gravemente amenazados de extinción, con las categorías IUCN de en peligro crítico (CR) o en peligro (EN), tales como Aquilegia saxifraga Casimiro-Soriguer Solanas \& Cabezudo (Sierra del Jobo, Málaga), Armeria grajoana Casimiro-Soriguer Solanas \& B. Cabezudo (Sierra de Alcaparaín, Málaga), Astragalus castroviejoi Talavera \& Sánchez Gómez (extremo norte de Almería y zonas aledañas de Murcia), Gadoria falukei Güemes \& Mota (Sierra de Gádor, Almería), Galatella malacitana Blanca, Gavira \& Suár.-Sant. (sierras de Alpujata, Aguas y Bermeja, Málaga), Linaria argillicola Juan, Blanca, Cueto, J. Fuentes \& L. Sáez (depresión del Guadiana Menor, límite provincial entre Granada y Jaén), L. becerrae Blanca, Cueto \& J. Fuentes (El Chorro, Málaga), Narcissus nevadensis subsp. herrerae Algarra, Blanca, Cueto \& J. Fuentes (Sierra Almijara, Granada), Ranunculus cherubicus subsp. girelae Fern. Prieto, Molero Mesa, Muñoz Díaz \& Sanna (Sierra Nevada, límite provincial entre Granada y Almería), Rivasmartinezia cazorlana Blanca, Cueto, Benavente \& J. Fuentes (Sierra de la Cabrilla, Jaén), o Vella pseudocytisus subsp. orcensis Vivero, Simón-Porcar, Pérez-Coll. \& Catalán (extremo norte de Granada), a los que hay que añadir muchas otras especies igualmente amenazadas que aún no se han incluido en el Catálogo Andaluz de Especies Amenazadas (BOJA, 2012) y que en total pueden ser más de un centenar. Es evidente que el esfuerzo por parte de las administraciones competentes en materia de protección de la flora es insuficiente, aunque desarrollan un buen trabajo con los escasos recursos económicos disponibles. Es necesario agilizar la actualización periódica de la Lista Roja y del Catálogo Andaluz, acorde con los rápidos avances en el conocimiento, de modo que permita dirigir los escasos recursos hacia plantas muy amenazadas que aún no se recogen en la legislación y que en muchos casos quedan fuera de cualquier figura de protección, tanto sobre la planta como sobre su hábitat.

\section{Agradecimientos}

A José E. Gutiérrez, coordinador del LIFE Olivares Vivos y delegado de SEO/BirdLife, por el apoyo y la información aportada para la redacción de este artículo, y a los agricultores del proyecto Olivares Vivos por el compromiso demostrado a favor de la biodiversidad del olivar.

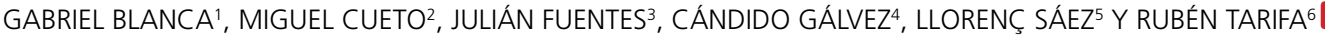
'Departamento de Botánica, Facultad de Ciencias, Universidad de Granada. ²Departamento de Biología y Geología, CECOUAL, Universidad de Almería. ${ }^{3}$ La Zubia, Granada. ${ }^{4}$ Semillas Silvestres, S.L. ${ }^{5}$ Unitat de Botànica, Facultat de Biociències, Universitat Autònoma de Barcelona. ${ }^{6}$ Departamento de Ecología Funcional y Evolutiva, Estación Experimental de Zonas Áridas, Almería.

\section{Bibliografía}

- Blanca G., B. Cabezudo, M. Cueto, C. Morales Torres \& C. Salazar (2011, eds). Flora vascular de Andalucía oriental. Universidades de Almería, Granada, Jaén y Málaga, Granada.

- BOJA (2012). Decreto 23/2012, de 14 de febrero, por el que se regula la conservación y el uso sostenible de la flora y la fauna silvestres y sus hábitats. BOJA 60: 114-163.

- IUCN (2012). IUCN red list categories and criteria ver. 3.1. IUCN Species Survival Commission.

- IUCN (2017). Guidelines for using the IUCN red list categories and criteria, ver. 13. IUCN Species Survival Commission.
- Rey P.J., A.J. Manzaneda, F. Valera, J.M. Alcántar, R. Tarifa, J. Isla, J.L. Molina-Pardo, G. Calvo, T. Salido, J.E. Gutiérrez \& C. Ruiz (2019). Landscape-moderated biodiversity effects of ground herb cover in olive groves: Implications for regional biodiversity conservation. Agriculture, Ecosystems \& Environment 277: 61-73.

- Sáez L. \& M. Bernal (2009) Linaria Mill. En: S. Castroviejo et al (eds), Flora iberica 13: 232-324. Real Jardín Botánico, CSIC, Madrid.
- Valdés B., S. Talavera \& E. Fernández-Galiano (1987). Flora vascular de Andalucía Occidental. Ketres, Barcelona. 